\title{
PANCREATITE AGUDA DEVIDA A HEMATOMA INTRAMURAL DO DUODENO POR USO DE ANTICOAGULANTE ${ }^{+}$
}

\author{
Samer FARHOUD*, Simone Moraes STEPHANI** e \\ Sansom Henrique BROMBERG***
}

\begin{abstract}
RESUMO - Racional - A hemorragia intramural espontânea do duodeno causada por complicações da terapêutica anticoagulante é rara e seu tratamento controverso. Objetivo - Apresentar a experiência advinda do tratamento de doente com essa condição clínica. Relato do caso - Expõe-se o caso de uma mulher de 71 anos de idade, que há 3 meses fazia uso de anticoagulante oral para tratamento de trombose venosa profunda dos membros inferiores. Apresentou-se com cefaléia e dores abdominais intensas no andar superior do abdome, associadas a náuseas e vômitos. Os exames laboratoriais e de imagem comprovaram o diagnóstico de surto agudo de pancreatite, decorrente de hematoma intramural de duodeno. Os valores de protrombina (49,7 s) e o sangramento de tecidos moles cervicais e urinário, sugeriam complicação da terapêutica anticoagulante. Resultados - A terapêutica conservadora foi efetiva, tendo a doente recebido alta, assintomática, no $10^{\circ}$ dia de internação. Conclusão - É recomendado o emprego do anticoagulante em doses menores nos doentes de risco e adequado controle dos parâmetros da coagulação. Acredita-se ser ideal a conduta conservadora e recomenda-se a cirurgia somente nos casos que evoluem com complicações.
\end{abstract}

DESCRITORES - Hematoma. Duodeno. Anticoagulantes. Hemorragia gastrointestinal. Pancreatite.

\section{INTRODUÇÃO}

Os hematomas intramurais de duodeno (HIMD) são raros e geralmente associados a traumatismo abdominal, sendo menos freqüentemente descritos em pacientes predispostos a distúrbios de coagulação ou que fazem uso de anticoagulantes. Mais raramente estão associados à presença de neoplasias pancreáticas, pancreatite aguda ou crônica, pâncreas ectópico, discrasia sangüínea, colagenoses e vasculopatias ${ }^{(3,6)}$.
O HIMD causa obstrução intestinal por compressão luminal e, quando o faz sobre a papila duodenal maior, pode determinar o aparecimento de icterícia e pancreatite. Atualmente, graças ao aperfeiçoamento e desenvolvimento dos métodos de imagens, há maior facilidade em se realizar o diagnóstico.

Nos últimos anos, a indicação do tratamento conservador na resolução desses hematomas vem substituindo com vantagens o procedimento cirúrgico, até então predominante. O presente estudo retrata aspectos ligados ao quadro clínico, diagnóstico e tratamento de uma paciente conduzida com sucesso.

\footnotetext{
+ Trabalho realizado na Pós-Graduação em Gastroenterologia Cirúrgica do Hospital do Servidor Público Estadual - Francisco Morato de Oliveira (HSPE-FMO).

* Pós-graduando de mestrado.

** Pós-graduanda de doutorado.

*** Vice-Coordenador do Curso de Pós-Graduação.

Endereço para correspondência: Dr. Sansom Henrique Bromberg - Rua Pedro de Toledo, 1800 - $1^{0}$ andar - sala 130 - Ibirapuera - $04039-004$ - São Paulo, SP. e-mail: depiamspe@uol.com.br
} 


\section{RELATO DO CASO}

Paciente do sexo feminino com 71 anos, branca, casada foi atendida no serviço de emergência, com queixa de cefaléia intensa há um dia, holocraniana, sem pródromos. Quatro horas depois, começou a apresentar dor abdominal localizada no epigástrio e mesogástrio, de início súbita, intensa, contínua, acompanhada de náuseas e vômitos biliosos. Referia sensação febril. Há três dias notara urina avermelhada, apresentando, desde então, disúria e polaciúria. Há 90 dias vinha fazendo uso de anticoagulante - varfarina sódica $5 \mathrm{mg}$ - uma cápsula ao dia, como tratamento de trombose venosa profunda e erisipela do membro inferior direito. Há 15 anos foi submetida a histerectomia por neoplasia. Negava passado de dor abdominal e traumatismos.

Ao exame, apresentava-se corada, discretamente desidratada, afebril. Notou-se extenso hematoma de orofaringe que progredia para as regiões distais, sendo o mesmo também observado na região lateral e anterior do pescoço. Reagia com dor de forte intensidade à palpação do epigástrio e mesogástrio. Ruídos hidroaéreos presentes, de timbre normal. O restante do exame físico não revelou anormalidades.

A avaliação neurológica e a tomografia computadorizada de crânio mostraram-se normais. A ultra-sonografia abdominal concluíu pela presença de esteatose hepática, com pâncreas no limite superior da normalidade, nódulo renal direito de $0,9 \mathrm{~cm}$ de diâmetro, aorta abdominal ateromatosa, mas de calibre normal. Chamou atenção extenso espessamento da terceira porção duodenal.

A tomografia computadorizada do abdome mostrou densificação moderada da gordura peripancreática, compatível com pancreatite aguda, e confirmou a presença de extenso espessamento parietal do duodeno, desde a porção descendente até o término da terceira porção, com afilamento luminal. As imagens eram sugestivas de hematoma intramural do duodeno e de pancreatite aguda (Figura 1).

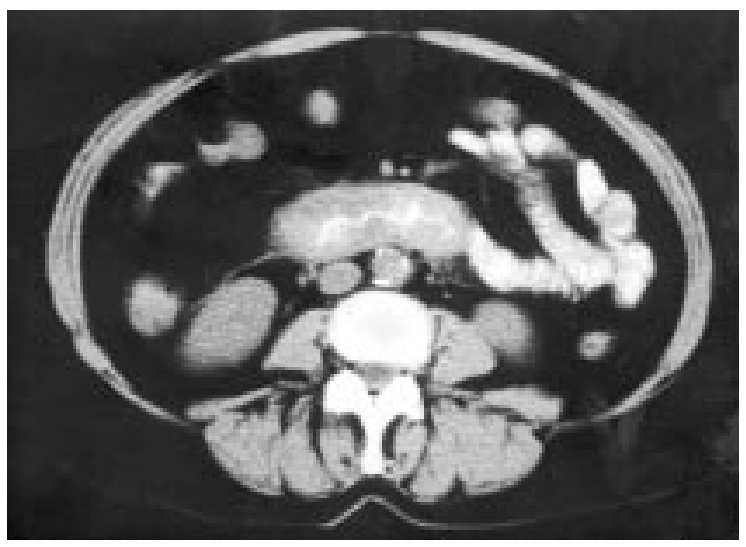

FIGURA 1 - Tomografia computadorizada do abdome utilizando contraste oral baritado, mostrando acentuado espessamento da parede duodenal em sua porção distal, com expressiva redução da sua luz
A esofagogastroduodenoscopia realizada no $7^{\circ}$ dia de internação, evidenciou mucosa duodenal de cor violácea, compatível com hematoma, e ausência de obstrução luminal (Figura 2).

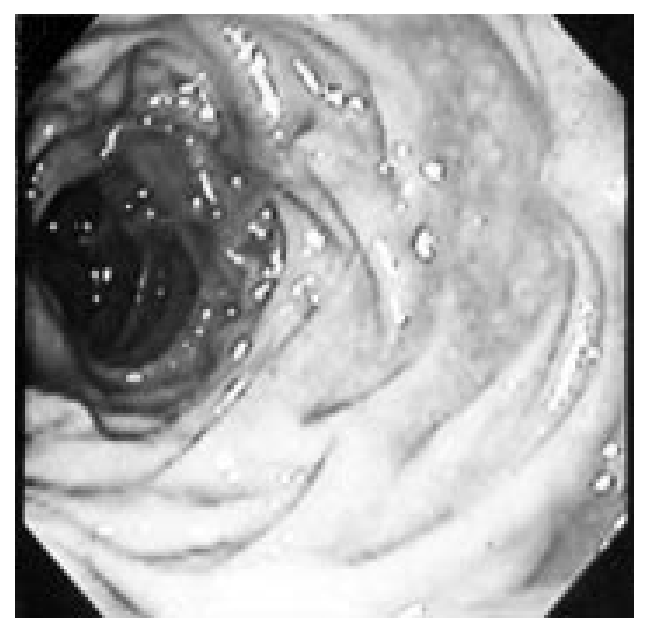

FIGURA 2 - Na porção distal da segunda porção do duodeno, notase acentuada alteração da coloração da mucosa, que progressivamente adquire aspecto típico de hematoma

O exame de urina tipo I mostrava a presença de hemoglobina +++ , leucócitos $624.000 \mathrm{cel} / \mathrm{mm}^{3}$, hemácias $396.000 \mathrm{cel} / \mathrm{mm}^{3}$. O hematócrito era de $40 \%$, notando-se leucocitose de $15000 / \mathrm{mm}^{3}$, com discreto desvio para esquerda e plaquetas normais. A atividade da protrombina era de $14 \%$ e o tempo de protrombina de $49,7 \mathrm{~s}$, fibrinogênio $501 \mathrm{mg}$ e a amilase era de 1562 u Somogyi.

No $4^{\circ}$ dia de internação, a amilase retornou aos valores normais, persistindo as alterações da protrombina e leucocitose. A bioquímica funcional do fígado não mostrava alterações. Notava-se baixa da hemoglobina ( $11,2 \mathrm{~g}$ ) e do hematócrito $(33 \%)$. No $5^{\circ}$ dia, a atividade da protrombina era de $70 \%$ e o tempo de protrombina de 14,9 s, que se normalizaram completamente no $6^{\circ}$ dia. A paciente apresentou boa evolução com o tratamento clínico, que consistiu da suspensão do anticoagulante oral, sonda nasogástrica, jejum, hidratação endovenosa, omeprazol $80 \mathrm{mg}$ endovenoso ao dia, fitomenadiona $10 \mathrm{mg}$ intramuscular ao dia e drogas sintomáticas. A dieta foi reintroduzida no $4^{\circ}$ dia, já com acentuada melhora da dor e dos exames laboratoriais. Recebeu alta no $10^{\circ}$ dia de internação; atualmente, 6 meses após, encontra-se bem, sem intercorrências.

\section{DISCUSSÃO}

O HIMD resulta da oclusão luminal do duodeno por hematoma devido à ruptura de vasos parietais. $\mathrm{O}$ seu principal agente etiológico é o traumatismo abdominal, mas em menor número de casos, o HIMD resulta de acidentes da terapia anticoagulante e de complicações das diáteses hemorrágicas, notadamente a hemofilia ${ }^{(2,4)}$. 
A formação do hematoma intramural pode ser observada em todos os segmentos do trato gastrointestinal, predominando naqueles de menor mobilidade, particularmente no duodeno (50\% a 73,3\%), devido a seus pontos extremos fixos, piloro e ligamento de Treitz. A sua localização retroperitonial em grande parte, a ausência de mesentério e o efeito da força traumática comprimindo-o contra a coluna vertebral, contribuem para essa maior incidência. A rica rede vascular que possui também é apontada como fator facilitador da hemorragia intramural ${ }^{(4)}$.

Classicamente, a lesão acomete os segmentos descendente (79,6\%) e transverso (72,2\%), como observado na doente em estudo, mas ocasionalmente pode envolver o bulbo e a primeira alça jejunal, sendo comum o comprometimento de vários segmentos. Os hematomas subserosos representam cerca de $65,6 \%$ dos casos, seguidos pelos submucosos $(31,2 \%)$ e musculares $(5,4 \%)^{(3)}$.

O hematoma cresce circunferencial e longitudinalmente, atingindo seu tamanho máximo entre 2 horas e 15 dias e produz obstrução por pressão. Proteínas originadas da desintegração da hemoglobina e da degradação tecidual, elevam a osmolaridade local e provocam o ingresso de fluidos que exercem a pressão obstrutiva luminal. Por vezes, chama atenção a ausência de coágulos, obviamente atribuída à ação da terapia anticoagulante, que deve ser rapidamente suspensa ${ }^{(2)}$.

Os anticoagulantes orais inibem a coagulação sangüínea por interferirem na síntese hepática dos fatores vitamina $\mathrm{K}$ dependentes (II, VII, IX e X), exercendo sua ação após 12 a 24 horas. Os níveis ideais no controle da anticoagulação correspondem a INR (International Normalized Ratio) de valor entre 2,0 e 3,0 e a uma atividade de protrombina entre $25 \%$ e $30 \%$. Os valores de protrombina encontrados na admissão da enferma sugerem hiperdosagem do anticoagulante, fator desencadeante do quadro clínico por ela apresentado.

A incidência anual de sangramento por cumarínicos é de $3 \%$ e a hemorragia pelo anticoagulante é 10 vezes maior no primeiro mês de uso $^{(7)}$. Os fatores de risco mais importantes são a idade ( $>65$ anos), a história de acidente vascular cerebral, a hemorragia digestiva e de doenças específicas concomitantes, como o infarto do miocárdio recente, insuficiência renal, fibrilação atrial, anemia intensa e hepatopatia cônica. Nesses doentes, o sangramento ou a hemorragia raramente ocorrem quando são utilizadas doses menores dos cumarínicos que, mesmo assim, mantêm sua eficácia antitrombótica ${ }^{(7)}$.

Os idosos constituem o grupo de risco mais exposto ao uso da anticoagulação e devem ser cuidadosamente acompanhados, quer no tocante ao controle laboratorial, quer quanto às drogas que consomem, por suas interações com os anticoagulantes. As que mais interagem com os cumarínicos são os diuréticos tipo tiazida e os antiinflamatórios não-hormonais: a aspirina, por sua ação antiplaquetária e os demais, por potencializarem a ação dos anticoagulantes.

O surto de pancreatite aguda pode ser causa ou conseqüência do HIMD. Quando grave, pode ocasionar a hemorragia intramural determinante do hematoma duodenal e quando este se instala por outras causas, é capaz de comprimir a papila duodenal maior, dificultando o fluxo pancreático e biliar, dando origem à icterícia e ao surto agudo de pancreatite ${ }^{(1)}$, explicação válida para a doente em estudo. Convém salientar que a icterícia também pode originar-se da reabsorção do sangue extravasado, assim como a febre e a leucocitose.

A dor de forte intensidade no abdome superior e os vômitos biliosos freqüentes são sintomas característicos, evidenciando a obstrução luminal desses doentes; esses sintomas também sugerem o surto agudo de pancreatite. Provavelmente, ambos os fenômenos contribuíram para a sintomatologia apresentada pela doente deste estudo. Febre, massa palpável (25\% a $75 \%)$ e rigidez muscular na porção superior do abdome são também freqüentes.

A história do consumo de anticoagulante, a presença de hemorragia em vários territórios orgânicos, a leucocitose, o aumento do tempo de protrombina, a hiperamilasemia e o achado de obstrução parcial do duodeno por imagem fazem o diagnóstico da afecção. A doente deste estudo apresentou fenômenos hemorrágicos indubitáveis da orofaringe, de partes moles cervicais e dos sistemas urinário e digestório, locais reconhecidos como os de sangramento mais freqüentes em virtude da ação indesejável dos anticoagulantes ${ }^{(7)}$.

A radiografia simples do abdome orienta no diagnóstico da síndrome obstrutiva alta, pelos sinais de dilatação gástrica e de escassa presença de ar no duodeno. $\mathrm{O}$ exame baritado mostra compressão da grande curvatura do estômago, acentuado edema das válvulas coniventes, assinalando com exatidão o local obstruído. Também evidencia o retardo do esvaziamento gástrico e defeitos intraluminares.

A endoscopia digestiva pode detectar eritemas e lesões purpúricas na mucosa gástrica e duodenal, que se torna violácea nas proximidades do hematoma, onde a luz reduzida não mais permite a passagem do aparelho. Este aspecto perdura por vários dias, como constatado na doente deste relato.

A tomografia computadorizada reconhece, inicialmente, o hematoma como massa de alta atenuação, assinalando com perfeição o grau de obstrução luminal e a extensão do processo mural. No caso presente, o estreitamento luminal era de vulto, mas, funcionalmente, resolveu-se a partir do $4^{\circ}$ dia de evolução. Também reconhece com facilidade as complicações do HIMD como os abscessos localizados e a perfuração ${ }^{(5)}$.

A conduta conservadora, pouco utilizada décadas atrás, é hoje a de eleição. A descompressão por meio de sonda nasogástrica, o uso de soluções parenterais, de vitamina $\mathrm{K}$ e eventual reposição de fatores da coagulação, aliados à observação cuidadosa, são medidas terapêuticas consideradas essenciais e de êxito, como comprovado. Eventualmente recorre-se à nutrição parenteral, que permite reposição calórica mais adequada e repouso alimentar, o que favorece a reabsorção do hematoma $^{(4)}$.

Embora o tratamento clínico mostre-se efetivo na maioria dos casos, existem condições que necessitam de indicação cirúrgica, 
tais como a hemorragia não-controlável, a necrose seguida ou não de perfuração e a estenose secundária. Alguns estudiosos aconselham a intervenção cirúrgica quando do aparecimento de sinais de peritonite ou caso não se verifique nítida melhora do quadro clínico após 7 a 10 dias de evolução ${ }^{(4,5,8)}$. A laparotomia, portanto, está reservada para as complicações do HIMD e, quando indicada, deve ser a menos agressiva possível, já que a evacuação do hematoma alivia o fenômeno obstrutivo e é adequada na maioria dos doentes, sendo excepcional a indicação de procedimentos cirúrgicos de maior porte ${ }^{(3,6)}$.

Farhoud S, Stephani SM, Bromberg SH. Acute pancreatitis due to intramural hematoma of the duodenum by use of anticoagulant therapy. Arq Gastroenterol 2001;38(1):53-56.

ABSTRACT - Background - Spontaneous intramural hemorrhage of the duodenum due to anticoagulant therapy is rare and the treatment is controversial. Objective - To present the acquired knowledge with the treatment of these disease. Case report - A 71-year-old women receiving for a 3 month period an anticoagulant therapy presented cervical bleeding of soft tissues and symptoms of acute pancreatitis and high small bowel obstruction. Early noninvasive diagnosis by computed tomographic scan was possible and conservative therapy proved successful in complete resolution of the pancreatitis and obstructive symptoms, with resumption of oral intake in the fourth day of treatment. The frequency of bleeding in high risk patients during warfarin therapy is reduced by less intense therapy, achieving a prothrombin time with an International Normalized Ratio of 2,0 to 3,0. Results - The use of conservative treatment was helpful and the patient was discharged asymptomatic, 10 days after admission. Conclusion - It is suggested conservative treatment for intramural hematoma of the duodenum and recommended laparotomy only when complications occur.

HEADINGS - Hematoma. Duodenum. Anticoagulants. Gastrointestinal hemorrhage. Pancreatitis.

\section{REFERÊNCIAS BIBLIOGRÁFICAS}

1. Davis DR, Thomas CY. Intramural hematoma of the duodenum and jejunum. Ann Surg 1961;153:394-8.

2. Fingerhut A, Eugène C, Pourcher J, Pelletier JM, Ronat R. Les hématomes intramuraux du duodénum. Gastroenterol Clin Biol 1980;4:144-8.

3. Fingerhut A, Rouffet F, Eugène C, Fendler JP, Hillion D, Ronai R. Nontraumatic intramural hematoma of the duodenum: report of 4 cases and review of the literature. Digestion 1983;26:231-5.

4. Gutstein DE, Rosenberg SJ. Nontraumatic intramural hematoma of the duodenum complicating warfarin therapy. Mt Sinai J Med 1997;64:339-41.
5. Jewett TC Jr, Caldarola V, Karp MP, Allen JE, Cooney DR. Intramural hematoma of the duodenum. Arch Surg 1988;123:54-8.

6. Jones WR, Hardin WJ, Davis JT, Hardy JT. Intramural hematoma of the duodenum. A review of the literature and case report. Ann Surg 1971;173:534-44.

7. Landefeld CS, Beyth RJ. Anticoagulant-related bleeding: clinical epidemiology, prediction and prevention. Am J Med 1993;95:315-28.

8. Touloukian RJ. Protocol for the nonoperative treatment of obstructing intramural duodenal hematoma during childhood. Am J Surg 1983;145:330-4.

Recebido em 16/5/2000. Aprovado em 17/8/2000. 